\title{
Pilot Study into Super-Fractionation Treatment Strategy of Acne and Rosacea*
}

\author{
Dirk Meyer-Rogge ${ }^{1}$, Ilja L. Kruglikov ${ }^{2}$ \\ ${ }^{1}$ Derma-Clinic, Karlsruhe, Germany; ${ }^{2}$ Wellcomet GmbH, Karlsruhe, Germany. \\ Email: dirk@meyer-rogge.de
}

Received June $4^{\text {th }}, 2013$; revised July $6^{\text {th }}, 2013$; accepted July $14^{\text {th }}, 2013$

Copyright @ 2013 Dirk Meyer-Rogge, Ilja L. Kruglikov. This is an open access article distributed under the Creative Commons Attribution License, which permits unrestricted use, distribution, and reproduction in any medium, provided the original work is properly cited.

\begin{abstract}
It is evident that matrix metalloproteinases (MMPs) are involved in the pathophysiology of different inflammatory skin diseases. In this pilot study, we investigated the ability of very high frequency ultrasound-frequencies of approximately $10 \mathrm{MHz}$ - to modulate the activity of MMPs in the skin in inflammatory skin diseases such as acne and rosacea. It was shown that such waves can significantly improve the skin appearance in these diseases; however, the results are dependent on applied treatment schedule. The best results were obtained through the application of a super-fractionation strategy in the form of home treatments carried out twice daily.
\end{abstract}

Keywords: Pilot Study; Acne; Rosacea; Super-Fractionation; Home Treatment

\section{Introduction}

Acne remains the most widespread dermatological problem, affecting almost all individuals at some point during adolescence or adult life. The conventional therapies provide various possible ways of controlling the disease in the majority of cases; however, they are often connected with some side effects (among them skin irritations, chelitis, photo sensibilisation, depression, dry eyes, leukopenia, etc.) and different well-known inconveniences for the patient. The remission time between breakouts averages several months, necessitating the multiple repetitions of courses of treatment.

The pathophysiology of acne is unclear, but it is generally believed that various factors are involved. It is well known that false anti-inflammatory therapy can cause the development of acne scars, which can lead to long lasting aesthetic and psychological problems. Thus, the development of new acne treatment methods, especially those with no or very limited side effects and can therefore be used repeatedly, is of great interest.

The theoretical basis for the development of a new treatment strategy comes from the recent cognition that matrix metalloproteinases (MMPs) are strongly involved

\footnotetext{
*Conflict of interests: Dr. I. Kruglikov is the managing partner of Wellcomet $\mathrm{GmbH}$. He was involved neither in clinical treatments nor in the assessment of treatment results.
}

in a pathophysiology of different inflammatory skin diseases, among them acne [1] and rosacea [2]. MMPs are zinc-dependent enzymes which play an important role in the physiological and pathological breakdown of connective tissue which is strongly modified in inflammatory skin. For example, the activities of MMP-1 (collagenase-1), MMP-3 (stromelisin-1) and MMP-13 (collagenase-3) are respectively 500, 1000 and 15 times higher in acne than in normal skin [1]. Similar MMP activation was observed in rosacea, eczema, psoriasis, etc.

Thus, a new treatment strategy for different inflammatory skin diseases, especially for acne and rosacea, could be a temporal reduction of specific MMPs' activities. However, effectiveness of this strategy must be strongly dependent on the relationship between the characteristic relaxation time of MMP reaction and treatment intervals, making the classical treatment schedules not evidently optimal. By making relaxation times of MMPs much smaller than the intervals between sequential treatments, every new treatment will confront the full relaxation of the tissue to its basement state. The opposite will occur in cases where the treatment intervals are smaller than the relaxation times of the MMP reaction, and one can expect the cumulative effect during treatment course. Consequently, the optimal treatment interval must theoreticcally exist and it has to be re-analysed from a viewpoint of the characteristic reaction time of a treated skin condi- 
tion to applied treatment method.

\section{Relaxation Times of Processes Involved in Extracellular Matrix Modulation}

Modulation of different MMPs is a non-specific reaction which was observed not only in different inflammatory diseases such as acne [1], rosacea [2], psoriasis [3], ulcus cruris [4,5], but also in some aesthetic skin problems, [6] as well as in UV-induced skin aging [7]. In many cases, the modulated MMPs are gelatinases (MMP-2 and -9) which can cleave collagen IV. Since collagen IV is the main structural component of basement membrane, this modulation can directly affect the integrity of the epidermal barrier. The potential role of epidermal barrier integrity in the pathophysiology of acne vulgaris was recently discussed in [8]. However, the relationship between epidermal barrier structure and skin condition looks to be much more general and evidently concerns different inflammatory skin diseases, making the original modulation of some specific MMPs in the skin to a nonspecific component in a pathophysiology of these diseases.

It can be assumed that every treatment method which restores the barrier integrity through reduction of MMP activity during some characteristic time must consequently improve the skin condition. This explains why one and the same method can be used for effective treatment of different skin inflammatory diseases. Conversely, this can also explain why different treatment methods can be effectively used for treatment of one and the same skin condition: this would be the case if such methods can differently but still effectively influence the MMPs' modulation. Different treatment modalities produce various characteristic relaxation times of MMP reaction, which can be casually responsible for a variation in treatment results.

Under normal physiological conditions, the characteristic relaxation time of a MMP reaction can be assessed to vary between several hours and 1 - 2 days [9], assumeing the optimal treatment interval is less than one day. Such treatment schedules are not usual in ambulant treatments, and their application will demand a new treatment strategy involving the patients in a treatment process.

In this study we have applied the strategy of superfractionation (application of reduced single treatment doses combined with significant increase of treatment frequency) for treatment of acne vulgaris and rosacea.

\section{Study Design and Methods Study Design}

This was a pragmatic, non-randomized, single-centre pilot study. Since it is well known that participants can correctly identify whether their treatment consisted of placebo or active US (see [6]), neither the participants nor the study nurses and the doctors were blinded.

\subsection{Subjects}

9 healthy subjects (8 females and 1 male) with mild to moderate facial acne vulgaris, as well as 3 healthy subjects ( 2 females and 1 male) with moderate rosacea, aged 15 to 63 years, finished the study (Table 1). The subjects who entered the study had diffusively scattered inflamematory or non-inflammatory lesions but no abscesses or cysts.

Exclusion criteria: subjects aged 12 years or younger; willingness to comply with study protocol; subjects with abscesses and cystic acne; subjects with other dermatological conditions which could affect the visual appearance of facial skin; widespread acne scarring; application of other anti-acne or anti-rosacea therapies (e.g. blue light, antibiotics, etc.) within 3 months; history of oral retinoid use within 1 year.

Every participant received the same standardised training into the technique of sonotrode movement. All study participants signed the written informed consent.

\subsection{Treatment Device}

The study was conducted with a new very high frequency ultrasound (VHF-US) device Skinova ${ }^{\circledR}$ (Wellcomet Gm$\mathrm{bH}$, Karlsruhe, Germany) operating with a frequency of approximately $10 \mathrm{MHz}$. This device consists of a programming station and several applicators (sonotrodes). The sonotrode can be programmed by doctor with a predefined program and needed number of allowed treatments using programming station. Every patient received one programmed sonotrode in a special case supplied with a charging station for a pre-determined period of time (normally 12 days) for home self-treatments. The

Table 1. Subjects and their treatment parameters.

\begin{tabular}{ccccc}
\hline Subject & Sex & Diagnosis & Sessions & Duration, days \\
\hline 1 & $\mathrm{f}$ & acne & 24 & 12 \\
2 & $\mathrm{f}$ & acne & 11 & 14 \\
3 & $\mathrm{f}$ & acne & 14 & 7 \\
4 & $\mathrm{f}$ & acne & 24 & 12 \\
5 & $\mathrm{f}$ & acne & 24 & 12 \\
6 & $\mathrm{f}$ & acne & 24 & 12 \\
7 & $\mathrm{f}$ & acne & 14 & 7 \\
8 & $\mathrm{f}$ & acne & 24 & 12 \\
9 & $\mathrm{~m}$ & acne & 10 & 12 \\
10 & $\mathrm{f}$ & rosacea & 24 & 12 \\
11 & $\mathrm{f}$ & rosacea & 22 & 12 \\
12 & $\mathrm{~m}$ & rosacea & 24 & 12 \\
\hline
\end{tabular}


investigator could control the number of treatments that were actually applied after the sonotrode was returned using the special service menu in the programming station. The patients were blinded to the information of such control possibility to avoid possible manipulation of the number of effectively applied sessions.

The maximum US intensity used in treatment programs for acne and rosacea was $0.5 \mathrm{~W} / \mathrm{cm}^{2}$; treatment time per program-10 min (for rosacea) and 12 min (for acne). To avoid the placebo effect, the ultrasound intensity was controlled for every sonotrode before the first treatment and after its return by digital ultrasound power metre UPM-DT-10 (Ohmic Instruments Co., Easton, MA, USA).

\subsection{Treatment Schedule}

All subjects were asked to carry out the treatments twice daily, with the number of sessions totalling between fourteen (during 7 days) and twenty four (during 12 days). The subjects were advised to treat the whole area with papules and pustules uniformly. Normal US contact gel was used as a coupling medium to avoid any additional pharmacological effects. Subjects were permitted to use the non-irritating facial cleanser before and after Skinova ${ }^{\circledR}$ treatment to remove the rest of gel. Subjects were also instructed to avoid the application of any other facial skin care products during the study.

\subsection{Clinical Assessment}

Subjects were evaluated at baseline, on the day of sonotrode return, and controlled 3 to 4 weeks later. Grading of skin condition severity was done using the standardised bilateral facial photographs.

Since only the subjects with facial acne and rosacea were involved in this study, the global acne grading system that also takes chest and back lesions into account could not be applied. Since both inflammatory diseases (acne and rosacea) were tested in the same study, we were also resigned to use the methods of lesion counting [10].

Assessment of the severity of facial acne and rosacea was done with the Leeds technique [11]. The scale of 0 (no acne lesions/skin redness) to 10 (most severe acne lesions/skin redness) was used for grading. The assessments at baseline and after treatment course were provided by subjects as well as by three independent dermatologists, two of which were blinded to treatment assignments. Because of a priori existing difference in absolute values of grading of the same condition done by subjects and dermatologists, only the percentage change of grading was used for true assessment of treatment effect. The statistical evaluation of these differences was done using the paired $t$-test.

\section{Results}

The non-placebo and non-systemic effect of this treatment was evidenced by face splitting; the effect was observed only on the treated side of the face. This confirms the previous results that we obtained by application of dual wave high frequency ultrasound in skin rejuvenation [6].

Scorings made by patients and by three doctors are presented in Table 2.

The basement patients' scoring was $6.58 \pm 0.86$. After the course of treatment this scoring was significantly reduced to $3.58 \pm 0.86(\mathrm{p}<0.05$, paired $t$-test), providing the patients' self-assessed skin improvement of $45.0 \pm$ $13.0 \%$. The basement doctors' scoring (for all three investigators) was $4.97 \pm 1.14$. After the course of treatment it was significantly changed to $2.69 \pm 0.78$ ( $\mathrm{p}<$ 0.05 , paired $t$-test), providing the average skin improvement assessed by all doctors of $45.6 \pm 10.6 \%$. Although there were some variations in absolute scoring between dermatologists (e.g. basement scoring of doctor \#1 was $5.17 \pm 1.28$, of doctor \#2-5.00 \pm 1.15 , of doctor \#3$4.75 \pm 0.92$ ), no statistically significant difference was observed in a grade of skin improvement between different doctors (42.3\%, 48.7\%, 45.8\%, correspondingly) which indicates the robustness of the applied scoring procedure.

Although the basement scoring significantly varied between patients and doctors, the assessment of skin improvement carried out by doctors was not statistically different from this of patients. This also supports the reliability of applied scoring procedure. No difference was found between the improvement grades in acne and rosacea patients.

Table 2. Treatment results.

\begin{tabular}{ccccc}
\hline Subject & $\begin{array}{c}\text { Patient } \\
\text { before/after }\end{array}$ & $\begin{array}{c}\text { Doctor \#1 } \\
\text { before/after }\end{array}$ & $\begin{array}{c}\text { Doctor \#2 } \\
\text { before/after }\end{array}$ & $\begin{array}{c}\text { Doctor \#3 } \\
\text { before/after }\end{array}$ \\
\hline 1 & $6 / 3$ & $5 / 3$ & $6 / 3$ & $5 / 2$ \\
2 & $8 / 5$ & $6 / 4$ & $5 / 4$ & $6 / / 5$ \\
3 & $5 / 4$ & $4 / 2$ & $4 / 2$ & $4 / 2$ \\
4 & $7 / 5$ & $5 / 3$ & $4 / 2$ & $4 / 2$ \\
5 & $7 / 3$ & $6 / 3$ & $5 / 3$ & $5 / 2$ \\
6 & $7 / 3$ & $3 / 2$ & $4 / 2$ & $5 / 3$ \\
7 & $7 / 3$ & $7 / 3$ & $6 / 3$ & $6 / 3$ \\
8 & $7 / 3$ & $7 / 3$ & $7 / 3$ & $6 / 3$ \\
9 & $5 / 3$ & $6 / 4$ & $6 / 3$ & $5 / 3$ \\
10 & $7 / 3$ & $5 / 3$ & $6 / 3$ & $4 / 2$ \\
11 & $7 / 5$ & $3 / 2$ & $4 / 2$ & $3 / 2$ \\
12 & $6 / 3$ & $5 / 3$ & $3 / 1$ & $4 / 2$ \\
\hline
\end{tabular}


Next we have proved whether the grade of skin improvement depends on the number of daily treatments. For this, we assumed there was no improvement without treatments during the observation period of 1 to 2 weeks. This assumption was supported by our preliminary bilateral experiments which demonstrated the local effect of VHF-US treatment with no improvement in a contralateral control site. There was a positive correlation between the grade of skin improvement and the number of daily treatments with coefficient of correlation of $r=$ 0.871 . This supports our main idea that an increase of treatment frequency over the usual values applied in classical ambulant treatment strategies can improve the treatment results. However, strict statistical validation of this statement demands a study with a bigger cohort of subjects.

Some examples of treatment results are presented in Figures 1-3.

Treatments with Skinova ${ }^{\circledR}$ had no side effects, were pain-free and well tolerated by patients. Subjects with rosacea claimed a short-termed erythema directly after the treatment which disappeared within one hour.

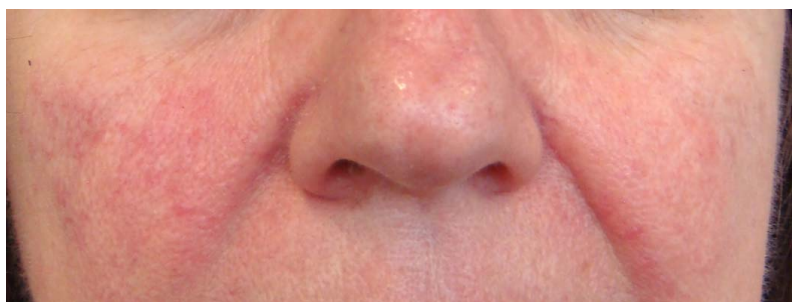

(a)

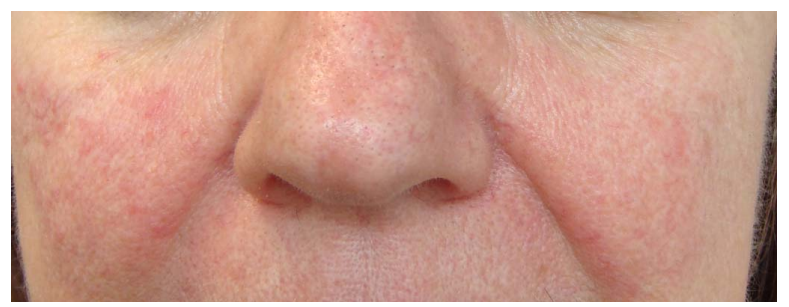

(b)

Figure 1. Rosacea: (a) before, (b) after 24 Skinova ${ }^{\circledR}$ home treatments in 12 days.

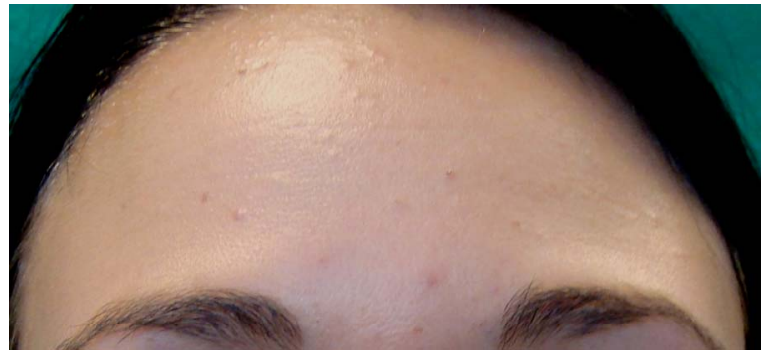

(a)

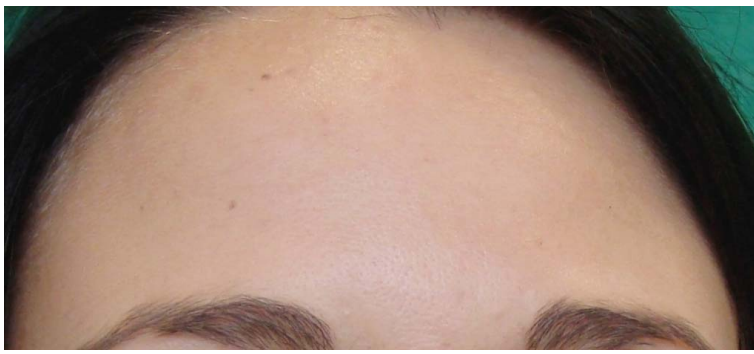

(b)

Figure 2. Acne seborrhoica: (a) before, (b) after 24 Skinova ${ }^{\circledR}$ home treatments in 12 days.

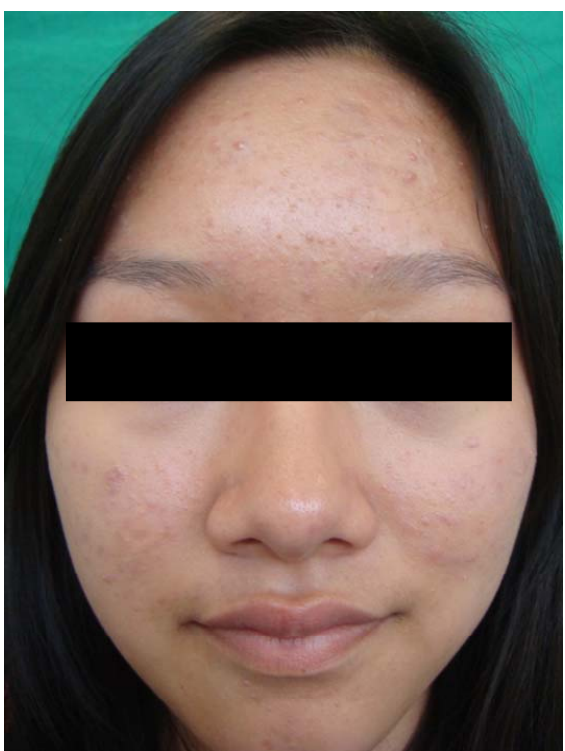

(a)

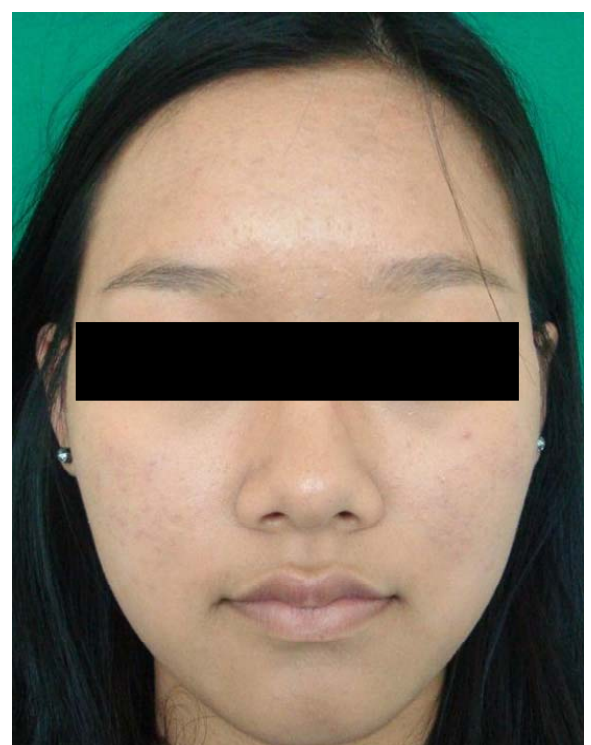

(b)

Figure 3. Acne: (a) before, (b) after 24 Skinova ${ }^{\circledR}$ home treatments in 12 days. 


\section{Discussion}

As demonstrated in our previous studies, VHF-US waves with frequencies about $10 \mathrm{MHz}$ alone or in combination with dual frequency US waves can be effectively used in some applications which primarily demand the improvement of connective tissue turnover, e.g. in treatments of non-healing wounds [5] or in aesthetic skin improvements [6]. The important feature of these US waves is their ability to produce a significant modulation of MMPs activity as well as of heat shock proteins [12]. Since the pathophysiologies of different inflammatory skin diseases are actually increasingly connected with the overexpression of some MMPs and destruction of the barrier skin function (especially that of the basement membrane), it was self-evident to apply this treatment method in the next step to such inflammatory skin conditions as acne and rosacea. However, since the relaxation times of MMPs after their modulation can be relatively short (normally less than 1 day), for such treatment to be effective it must be connected with the application of a special treatment schedule which demands a high (up to twice daily) frequency of treatments. We named this treatment schedule "super-fractionation".

Our results demonstrate a significant skin improvement in acne and rosacea patients after application of VHF-US in a super-fractionated schedule. The observed grade of skin improvement in acne and rosacea patients in this study reached in average almost $50 \%$ after one to two weeks of regular treatments. Although a similar grade of acne skin improvement was observed in application of other treatment modalities, an unusually short ( 1 - 2 weeks) treatment time was needed to see this improvement. We can conclude that VHF-US can be principally used for effective treatment of at least some inflammatory skin diseases. Similar improvement of skin condition in acne and rosacea through application of one and the same modality using the same treatment schedule must evidently mean the involvement of the same pathophysiological step. It is quite likely that it is due to the modulation of specific MMPs' activity, which is normally significantly increased in both diseases.

Since only two subjects deviated from the proposed treatment schedule and applied the VHF-US treatments less than once daily (subjects \#2 and \#9) whereas the majority of patients used the super-fractionation strategy with two treatments per day, it was not possible to make the full statistical analysis for the dependence of skin improvement on a treatment schedule. While the observed correlation of 0.871 between skin improvement and number of daily treatments points to a better skin reaction by regular applications, this important aspect must be investigated further in a special cohort of subjects under controlled variation of the total number of treatments as well as of a treatment frequency.
Another open question of this pilot study remains the remission time after application of super-fractionation treatment strategy. Although the obtained results were stable at the moment of a controlled investigation $3-4$ weeks after the treatment course, a much longer followup will be needed to make the quantitative prognosis.

\section{Conclusion}

The present pilot study has demonstrated that the application of VHF-US of approximately $10 \mathrm{MHz}$ can significantly improve a skin condition in acne and rosacea. The schedule of super-fractionation with application of treatments twice daily during one to two weeks appears to be optimal for such indications. A controlled study will be needed in order to obtain reliable information concerning the remission time after such intensive treatment courses, to define the optimal treatment schedule and to carry out the statistical analysis of long term results.

\section{REFERENCES}

[1] S. Kang, S. Cho, J. H. Chung, C. Hammerberg, G. J. Fisher and J. J. Voorhees, "Inflammation and Extracellular Matrix Degradation Mediated by Activated Transcription Factors Nuclear Factor- $k B$ and Activator Protein-1 in Inflammatory Acne Lesions in Vivo,” American Journal of Pathology, Vol. 166, No. 6, 2005, pp. 1691-1699. doi:10.1016/S0002-9440(10)62479-0

[2] R. R. Bonamigo, L. Bakos, M. Edelweiss and A. Cartell, "Could Matrix Metalloproteinase-9 Be a Link between Demodex Folliculorum and Rosacea?” Journal of the European Academy of Dermatology and Venerology, Vol. 19, No. 5, 2005, pp. 646-647. doi:10.1111/j.1468-3083.2005.01221.x

[3] R. Fleischmajer, K. Kuroda, R. Hazan, R. E. Gordon, M. G. Lebwohl, A. N. Sapadin, F. Unda, N. Iehara and Y. Yamada, "Basement Membrane Alterations in Psoriasis Are Accompanied by Epidermal Over Expression of MMP-2 and Its Inhibitor TIMP-2,” Journal of Investigative Dermatology, Vol. 115, No. 5, 2000, pp. 771-777. doi:10.1046/j.1523-1747.2000.00138.x

[4] R. Lobmann, A. Ambrosch, G. Schultz, K. Waldmann, S. Schiweck and H. Lehnert, "Expression of Matrix-Metalloproteinases and Their Inhibitors in the Wounds of Diabetic Patients,” Diabetologia, Vol. 45, No. 7, 2002, pp. 1011-1016. doi:10.1007/s00125-002-0868-8

[5] I. Kruglikov and E. Kruglikova, "Dual Treatment Strategy by Venous Ulcers: Pilot Study to Dual-Frequency Ultrasound Application," Journal of Cosmetics, Dermatological Sciences and Applications, Vol. 1, No. 4, 2011, pp. 157-163. doi:10.4236/jcdsa.2011.14024

[6] D. Meyer-Rogge, F. Rösken, P. Holzschuh, B. D’hont and I. Kruglikov, "Facial Skin Rejuvenation with High Frequency Ultrasound: Multicentre Study of Dual-Frequency Ultrasound," Journal of Cosmetics, Dermatological Sciences and Applications, Vol. 2, No. 2, 2012, 
pp. 68-73. doi:10.4236/jcdsa.2012.22016

[7] T. Quan, Z. P. Qin, W. Xia, Y. Shao, J. J. Voorhees and G. J. Fisher, "Matrix-Degrading Metalloproteinases in Photoaging," Journal of Investigative Dermatology. Symposium Procedures, Vol. 14, No. 1, 2009, pp. 20-24. doi:10.1038/jidsymp.2009.8

[8] D. Thiboutot and J. Q. Del Rosso, “Acne Vulgaris and the Epidermal Barrier. Is Acne Vulgaris Associated with Inherent Epidermal Abnormalities that Cause Impairment of Barrier Functions? Do Any Topical Acne Therapies Alter the Structural and/or Functional Integrity of the Epidermal Barrier?” The Journal of Clinical and Aesthetic Dermatology, Vol. 6, No. 1, 2013, pp. 18-24.

[9] G. J. Fisher, S. C. Datta, H. S. Talwar, Z. Q. Wang, J. Varani, S. Kang and J. J. Voorhees, "Molecular Basis of Sun-Induced Premature Skin Ageing and Retinoid An- tagonism,” Nature, Vol. 379, No. 6563, 1996, pp. 335339. doi:10.1038/379335a0

[10] B. Adityan, R. Kumari and D. M. Thappa, "Scoring Systems in Acne Vulgaris," Indian Journal of Dermatology, Venerology and Leprology, Vol. 75, No. 3, 2009, pp. 323326. doi:10.4103/0378-6323.51258

[11] B. M. Burke and W. J. Cunliffe, "The Assessment of Acne Vulgaris: The Leeds Technique,” British Journal of Dermatology, Vol. 111, No. 1, 1984, pp. 83-92. doi:10.1111/j.1365-2133.1984.tb04020.x

[12] W. Sontag and I. Kruglikov, "Expression of Heat Shock Proteins after Ultrasound Exposure in HL-60 Cells," Ultrasound in Medicine and Biology, Vol. 35, No. 6, 2009, pp. 1032-1041. doi:10.1016/j.ultrasmedbio.2008.12.011 